\title{
An Efficient HCF Scheduling Mechanism in Mixed Traffic Scenarios
}

\author{
David Gozálvez ${ }^{1}$, Jose F. Monserrat ${ }^{1}$, Javier Gozálvez ${ }^{2}$ and Lorenzo Rubio ${ }^{1}$ \\ ${ }^{1}$ Polytechnic University of Valencia (UPV) - Mobile Communications Group, Spain \\ ${ }^{2}$ University Miguel Hernández - Signal Theory and Communications Division, Spain \\ dagoser@teleco.upv.es, jomondel@dcom.upv.es,j.gozalvez@umh.es, lrubio@dcom.upv.es
}

\begin{abstract}
In this paper an efficient scheduling mechanism for wireless local area networks (WLAN) is proposed. This innovative scheme, named hybrid HCCA-EDCA centralised scheme (HHECS), is based on the Hybrid Coordination Function (HCF) protocol introduced by the IEEE 802.11e standard. The HHE-CS aims at satisfying the user quality of service $(\mathrm{Q} o S)$ demands for real time (RT) services with variable bit rate while maximising the throughput of best effort users. This paper proves conclusively that an adequate allocation of downlink and uplink traffic between EDCA and HCCA increases system performance as compared with more traditional schemes in which all best effort traffic is exclusively delivered by a distributed mechanism like EDCA.
\end{abstract}

Keywords- Wireless LAN, HCCA, EDCA, Scheduling

\section{INTRODUCTION}

In last years the use of WLANs has become widespread in the high bit rate wireless access market, especially in indoor environments. At the same time, real time (RT) multimedia services, such as voice over IP or video streaming, are increasing their popularity in such a way that WLANs are beginning to be considered as one more Radio Access Technology (RAT) inside Heterogeneous Wireless Networks [1]. Therefore, a MAC Protocol capable of providing QoS has to be devised in order to guarantee a similar performance to the one offered by packet-based cellular systems as GPRS or HSDPA.

Although the standard 802.11 has become a de facto standard for WLAN, it was originally unable to satisfy any level of QoS and therefore to transport any type of RT service. Because of this, the standard IEEE 802.11e was ratified at the end of 2005 to allow the provision of QoS in WLAN networks [2]. In the IEEE 802.11e standard a new Hybrid Coordination Function (HCF) protocol is specified to extend the classical DCF (Distributed Coordination Function) and PCF (Point Coordination Function) of the previous legacy IEEE 802.11 MAC. The HCF defines two channel access methods, namely EDCA (Enhanced Distributed Channel Access), distributed and contention-based, and HCCA (HCF controlled channel access), centralised and contention-free.

In the literature there are quite a few of contributions studying separately the performance of HCCA and EDCA. These studies demonstrate, either analytically or via simulation, the capability of HCF to protect real time services from the rest of traffic in the network, and at the same time fulfil their delay and throughput requirements [3], [4]. Regarding multimedia traffic, in the literature there is not an agreement in relation to which channel access mechanism is better suited to serve it. Better than choosing only one channel access mechanism, some authors have stated that an adequate combination of HCCA and EDCA is the best solution and offers the highest performance [4]. In this paper a novel scheduling scheme called Hybrid HCCA-EDCA centralised scheme (HHE-CS) is proposed seconding this last motion. The HHE-CS alternates EDCA and HCCA following a specific policy in such a way that it outperforms previous scheduling mechanisms and is the best suited to transport both RT and best effort traffic.

The paper is organised as follows. In Section II the new MAC protocol of the IEEE 802.11e standard is described paying special attention to the two new channel access mechanisms. Section III is devoted to explain in full detail the new HHE-CS scheduling scheme. In Section IV the employed simulation tool, as well as the simulation scenario, are presented. Finally the obtained results are discussed in Section V whereas Section VI concludes.

\section{Channel Access Mechanisms In IEEE 802.11E}

The new MAC protocol HCF is the principal addition of the IEEE $802.11 \mathrm{e}$ to the legacy 802.11 standard. This protocol satisfies the QoS required by RT services by means of two different channel access mechanisms that must be implemented in all the 802.11e compatible devices: EDCA and HCCA. The HCF is able to alternate contention-based EDCA channel access periods with polling-based HCCA channel access periods.

In EDCA, like in DCF, each station, including the access point (AP), has to content to gain control of the medium in order to transmit one data frame. Before transmitting, an active station has to wait a random number of time slots after sensing whether the channel is free for a time duration called DCF inter-frame space (DIFS). The number of waiting slots is randomly chosen between 0 and a contention window $(\mathrm{CW})$ that starts with a value of CWmin. Every time that a frame is not positively acknowledged because of either a channel error or a collision, the $\mathrm{CW}$ is doubled until it reaches a value called CWmax. 
EDCA access method enhances the DCF contention mechanism to guarantee prioritised QoS thanks to a specific service priority policy. This way, more delay-sensitive services are served with a higher statistical probability than best effort services, providing what is usually referred to as soft QoS. This is accomplished due to the fact that in EDCA the contention mechanism can be particularised for each of the four different access categories (AC) defined by the standard, namely voice, video, best effort and background. The parameters that differentiate each $\mathrm{AC}$ are: the arbitration interframe space (AIFS), the CWmin, the CWmax and the transmission opportunity (TXOP). The concept of TXOP is an important new feature of the IEEE 802.11e standard. A TXOP is a time interval in which a given station has the medium control to send packets in burst mode without having to content for the medium.

On the contrary, HCCA is a centralised channel access mechanism based on a higher priority of the AP to achieve the channel control thus distributing the resources in a controlled manner. With HCCA it is possible to ensure a user-specific QoS distinguishing each user state; this is the so-called hard QoS. HCCA is quite similar to the legacy PCF protocol except for the fact that with the new standard the AP can take control of the medium not only during the contention-free periods (CFP), but also during the contention periods (CP), setting up controlled access phases (CAP). During one CAP, the AP, applying a certain polling scheme, either allocates the medium to uplink stations to transmit during a specific TXOP time or serves itself downlink traffic.

The IEEE 802.11e standard defines a reference schedule for HCCA in which every service interval time (SI) the AP polls every admitted station allowing them to transmit uplink traffic during a previously calculated TXOP time. The SI and the duration of each TXOP are calculated depending on the service requirements of the associated stations in order to satisfy their different levels of QoS. Figure 1 shows this reference schedule.

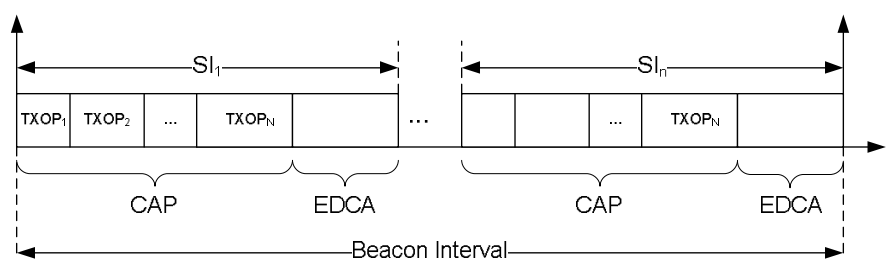

Figure 1. Structure of the IEEE 802.11e Beacon Interval

Although both channel access mechanisms provide a certain level of QoS not available in the previous legacy IEEE 802.11, several studies as [4] indicate the inability of EDCA to fulfil the RT requirements when the number of stations increases. Besides, in distributed systems like DCF or EDCA, every station of the same $\mathrm{AC}$, including the $\mathrm{AP}$, has the same probability of gaining control of the medium. Due to this fact, and because it is normal for the AP to have a lot more information pending than any other station in the system, in [5] it was demonstrated that an important downlink bottleneck will degrade the global throughput of the network affecting all kind of services. Despite the fact that the 802.11e standard allows defining a specific set of contention parameters for the AP different from the rest of the stations in the system, the tuning of these parameters to achieve the desired system behaviour can be a very complicated task that in most cases offers not enough differentiation.

Since EDCA is not able to satisfy completely the QoS requirements of $\mathrm{RT}$ services, $\mathrm{HCCA}$ is the preferred option for this type of traffic. The polling scheme defined in the standard for HCCA presents serious deficiencies when variable bit rate traffic (VBR) is considered since the AP is not able to modify the TXOPs duration according to the instantaneous variable bit rate. Some studies as [6] propose the use of traffic-aware dynamic algorithms to adapt each SI the length of all the TXOPs to the size of the users' buffer. However, this paper will show that in case of bidirectional traffic, the problem of handling VBR services can be solved without using such techniques, allowing the admission of the same number of stations than in the constant bit rate (CBR) case and guaranteeing the required QoS.

Besides, the HCCA polling mechanism introduces an overhead in the network due to the polling frames that indicate the beginning and ending of a polled TXOP. This overhead must be considered since it reduces HCCA efficiency when handling uplink traffic.

The new scheduling scheme proposed in this paper called HHE-CS takes into account all the advantages and disadvantages of both HCCA and EDCA mechanisms alternating them in an optimal manner.

\section{HHE-CS}

\section{A. HHE-CS with RT Traffic}

If WLAN is integrated within a heterogeneous network, RT services, as for instance voice over IP (VoIP) or video telephony, will be handled jointly with best effort services. As it is of paramount importance to satisfy the QoS of RT users, the use of HCCA is the preferred option due to its capability of offering guaranteed QoS. In consequence, HHE-CS transmits all the RT traffic through HCCA. However, due to the previously-mentioned inefficiencies, to achieve the same QoS for VBR traffic as for CBR, the classical approach of allocating time to stations on the basis of the mean sending rate is no longer valid. In HHE-CS an extra time is allocated to each user to support any sudden increase in data rate that can overflow the assigned TXOP. Due to this reservation of a bigger amount of time for each station, system capacity could seem to be reduced since, to some extent, a portion of the TXOP is wasted when data rate decreases and there are not enough bits in the buffer to make use of all the time assigned to the user. However, if bidirectional traffic is taken into account, the new HHE-CS uses the free time reserved for the uplink traffic to transmit also downlink data. This way, HHECS takes advantage of the higher capacity needed by VBR services and is able to maintain system capacity while fulfilling the QoS requirements. A robust call admission control (CAC) assures that the number of RT stations in the network never exceeds its maximum capability. To this end, the CAC denies the access to any station when the system is 
saturated. The number of RT stations that the HHE-CS CAC can admit in the system is directly proportional to the portion of time of the SI reserved for contention free transmissions in the network. Although the uplink RT stations are polled in a strict Round Robin (RR) scheme, the same is not necessarily true for downlink transmissions. In order to distribute more efficiently the downlink frames among RT users, different scheduling algorithms can be employed. In this paper, RR and First Come First Served (FCFS) algorithms are investigated when serving downlink RT traffic through HCCA. In the RR scheduling the AP transmits sequentially one frame to the last served station that has pending data. On the contrary, FCFS always transmits information corresponding to the oldest packet received by the MAC.

\section{B. HHE-CS with Best Effort Traffic}

Since best effort traffic has not strict constraints to be fulfilled, it is better suited for EDCA transmission because of the inefficiency of the polling system of HCCA in the uplink. However, this limitation does not hold good in downlink transmissions where the AP knows perfectly the state of every buffer and can send data directly to a station without the necessity of transmitting a polling frame first.

CAC only accepts an incoming RT user if its QoS is guaranteed even in the worst case scenario, i.e. when the system is saturated and all stations transmit at their minimum negotiated bit rate. As usually the system is not congested or the stations transmit at a faster data rate than the minimum, some bandwidth will not be used. This free time, in addition to all the time allocated to the uplink VBR traffic wasted by RT services, will be employed by the HHE-CS mechanism to solve some of the disadvantages of EDCA. Thanks to its totally controlled access scheme, HHE-CS takes advantage of the HCCA ability to seize the medium before any other station in order to transmit, during the remaining CAP time, best effort traffic. The distribution of the remaining time among the best effort users will be carried out according to a specific scheduling algorithm implemented in the AP. To maximise the network best effort throughput, the amount of data transmitted to each station must be proportional to the capacity of transmission of the user, allowing the fastest stations to receive information at a faster bit rate without being degraded by slower stations. With this aim, HHE-CS employs a novel scheduling algorithm based on tokens in which the AP transmits more often to stations that are in a better channel condition and are working in a higher transmission mode. In this algorithm each station gains tokens at a rate proportional to their transmission rate so that the AP only has to choose the station with more tokens in order to send him the next frame. Every time a station receives successfully a frame, a fixed number of tokens is subtracted to this station. In order to assess the performance of the novel scheduling algorithm, other schedulers have been implemented: RR, FCFS and Maximum Carrier to Interference Ratio (MaxCIR) allocation. With FCFS the objects of the different web pages are transmitted entirely in their order of arrival, and the MaxCIR scheduler always transmits to the station with better channel conditions thus maximising the network throughput.

To prevent transmissions of best effort traffic from jeopardising the performance of RT traffic, RT users have always higher priority over best effort users in the downlink scheduling with HCCA. This way, the QoS of RT communications will remain practically constant regardless how many best effort stations are transmitting in the network. Contrary to the already defined CAC for RT users, not any admission management has been implemented for best effort services, i.e. any best effort user asking for service will be accepted in the system.

The rest of downlink best effort traffic not transmitted by HCCA and all the uplink best effort traffic are served in the next contention-based period through EDCA. This choice has been made since distributed channel access mechanisms are more efficient when transmitting uplink traffic. Also, to greater enhance the performance of EDCA, the CWmin value has been tuned to give more priority to the AP than the other stations, as recommended in [5].

\section{EVALUATION ENVIRONMENT}

To conduct this investigation an evolved version of the emulator presented in [7] has been employed. This tool is capable of emulating a heterogeneous wireless network constituted by several cellular and wireless systems such as GPRS, EDGE, HSDPA and of course WLAN in the form of the 802.11 standard. More specifically, both $802.11 \mathrm{~b}$ and 802.11g physical layers have been implemented as well as the MAC protocol specified by the 802.11e extension. In the simulations presented in this paper all the RATs except WLAN have been disabled to ensure that they have not any kind of influence in the obtained results.

In the simulator Web traffic has been modelled as best effort traffic sources. The web browsing service has been implemented as proposed in [8]. The mathematical distributions have been parameterised to match an average throughput per user of $60 \mathrm{kbps}$ in downlink and $10 \mathrm{kbps}$ in uplink.

Real-time services have been included via the emulation of real-time H.263 video following the model presented in [9]. This model employs the VBR H.263 codec that generates instantaneous changes in the output bit rate while maintaining an average constant bit rate of $64 \mathrm{kbps}$. Opposed to the Web traffic model, in the H.263 model, the traffic source does not wait to the completion of the transmission of the last video frame before generating the next one. In this case, the station assumes that the QoS requirements of the video service have not been fulfilled and the older frame is discarded. Because of this, a very important parameter to evaluate the QoS of H263 users is the percentage of frames discarded throughout the simulation, a parameter that in this paper is called user equipment satisfaction (UeS). A UeS threshold of $95 \%$ is set in the scheduler design as the minimum QoS level that must be reached by the WLAN in order to consider that RT services are being served with enough quality. 


\section{RESULTS}

First of all, to evaluate the performance of HHE-CS with RT H.263 VBR traffic and to assure that the proposed scheduler is able to provide the required QoS, the worst case scenario is studied where all stations are transmitting at the lowest data rate of 6 Mbps. Due to the non-constant nature of the H.263 codec, the TXOP reserved to each station has to be increased. Performed simulations show that an increase of about a $100 \%$ as compared with the CBR case is needed in order to obtain UeS values over the established $95 \%$ threshold. This increase in the time allocated to each station should force the CAC to admit half the users in the system when the reference scheduler is employed. However, Fig. 2 shows the capability of HHE-CS to maintain the QoS level of VBR H263 bidirectional users and at the same time achieving the same capacity as with CBR users which is of 25 bidirectional users. The two previously discussed scheduling algorithms for RT downlink transmission are investigated, i.e the RR based scheduling and the FCFS based scheme. The results show a slightly better UeS when using the FCFS algorithm although both solutions offer enough guarantees of QoS.

Next, the behaviour of HHE-CS with best effort traffic is studied analysing the improvement in total throughput with respect to the more traditional approach. The cell WWW throughput obtained using HHE-CS and a scheme where all best effort traffic is transmitted through EDCA is compared. A fixed number of $25 \mathrm{H} 263$ bidirectional users are simulated whereas the number of WWW users varies from 10 up to 60 . The time reserved for contention free transmissions is fixed at the $90 \%$ of the time. Also, to reveal the reduction in the downlink bottleneck occasioned by contention transmissions, the ratio between the downlink WWW throughput and the uplink WWW throughput is calculated. It is worth noting that, since the traffic generated by downlink WWW users is in average six times higher than uplink users, this ratio is expected to be around 6 . All stations are transmitting at 6 Mbps. As it can be seen in Fig. 3 and Fig. 4 both schemes have similar throughputs until the network reaches the saturation point from which the HHE-CS not only achieves an overall better throughput but also manages to maintain a higher $\mathrm{DL} / \mathrm{UL}$ ratio. This is possible due to the fact that when transmitting downlink traffic through a distributed mechanism, an important part of the time is wasted during contention whereas HCCA can seize the channel and start transmitting almost immediately. Also, since downlink transmissions through HCCA are made by the AP without the contention of the uplink stations, the DL/UL ratio can be greatly improved as it is shown in the results. It can also be noted that once the number of station surpasses the saturation point, the cell performance decreases because of the increasing number of collisions that take place in the system. Although the HHE-CS achieves an important improvement in the DL/UL ratio, this is not enough to assure an adequate balance between uplink and downlink traffic. To achieve this objective, further increments of the time reserved for contention free transmissions can be made.

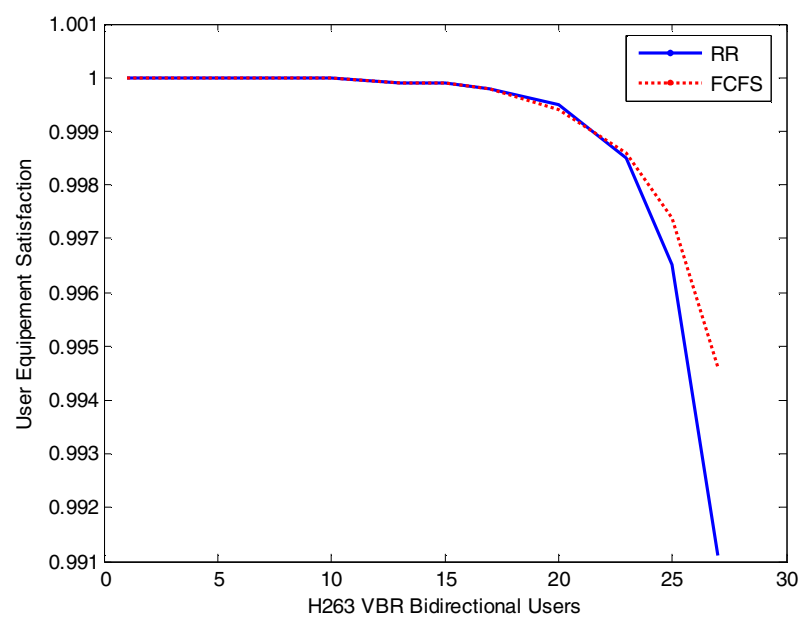

Figure 2. User Satisfaction with bidirectional traffic

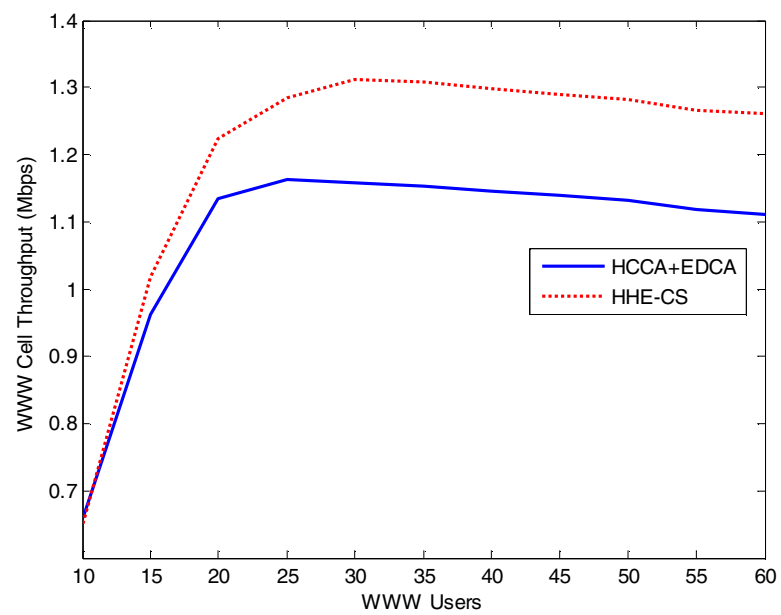

Figure 3. WWW Cell throughput for HHE-CS and HCCA+EDCA with an increasing number of WWW users

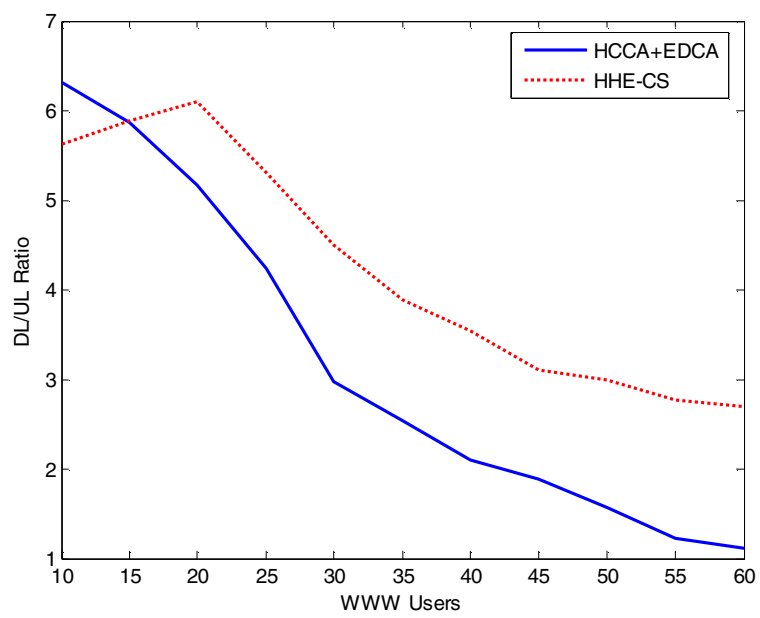

Figure 4. DL/UL ratio for HHE-CS and HCCA+EDCA with an increasing number of WWW users 
Finally, the total cell throughput and user throughput obtained with different scheduling algorithms are compared. In these simulations, the AP transmits to $40 \mathrm{WWW}$ users via HCCA in downlink during only the $0.1 \%$ of the CAP time in order to saturate the system. Besides, no H263 stations are admitted in the network to focus the results in the best effort scheduling. The stations are now transmitting at a bit rate adequate to their distance to the AP. Fig. 5 and Fig. 6 show how the RR is the more constant algorithm along the SI simulated and among the users, although it fails in achieving the maximum network performance since the AP is transmitting approximately the same number of frames to each station without taken into account its transmission rate. As it can also be observed, the MaxCIR algorithm along with the Token based scheduler penalises the slower stations in order to transmit more often to the faster ones. However, in this scenario the Tokens based algorithm is able to ensure a minimum throughput of $15 \mathrm{kbps}$ to the slower stations whereas the MaxCIR algorithm only allocates 4 kbps as shown in Fig 6. The Tokens-based algorithm avoids the starvation of the stations operating in lower transmissions rates.

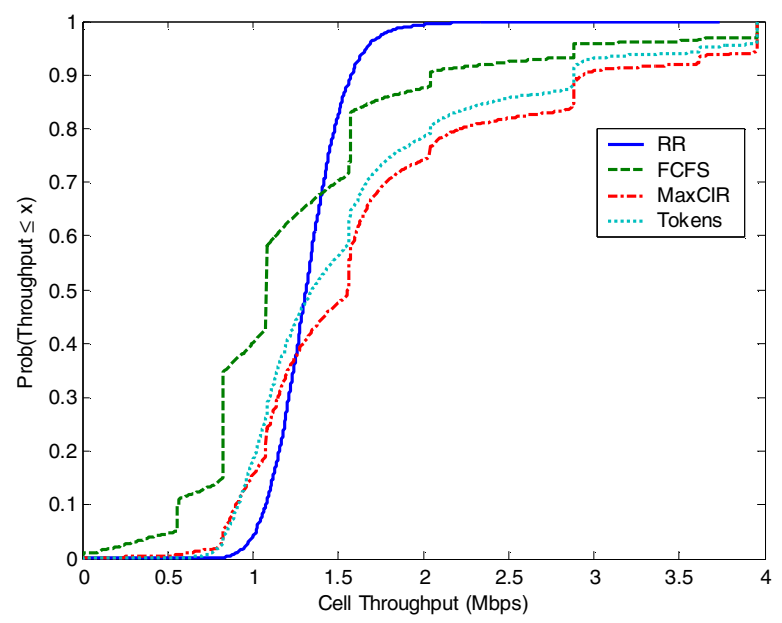

Figure 5. CDFs of the WWW Cell throughput of the four different scheduling algorithms considered for best effort traffic

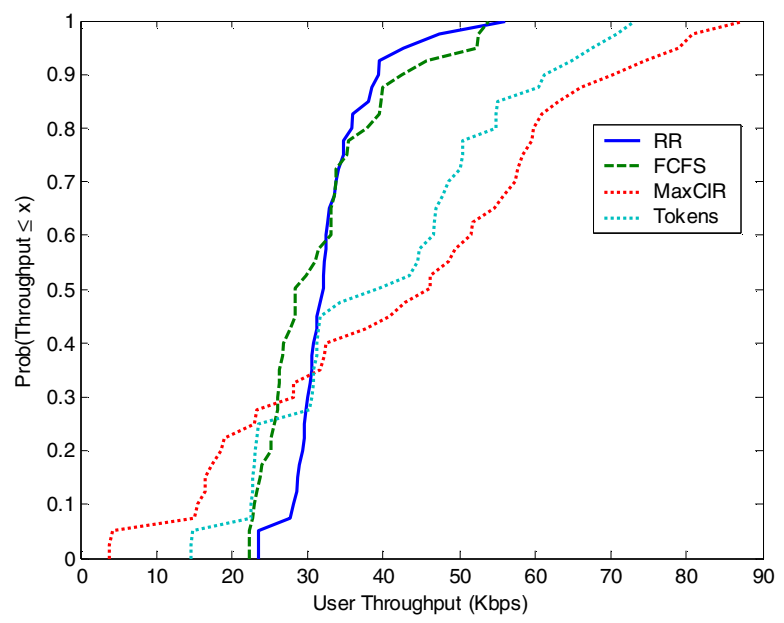

Figure 6. CDFs of the WWW User throughput of the four different scheduling algorithms considered for best effort traffic

\section{CONCLUSIONS}

In this paper a new scheduling scheme named HHE-CS that makes jointly use of HCCA and EDCA has been presented. Contrarily to more traditional philosophies in which best effort traffic is transmitted in a contention manner, HHE-CS takes advantage of the higher priority of HCCA to seize the channel and to send as much downlink best effort traffic as possible in the contention-free period, solving in this way the downlink bottleneck. As HHE-CS only employs the time not used by RT traffic, it is able to improve the performance of best effort traffic without compromising the quality of real time services. Some preliminary results have pointed out that a dynamic reservation of the CAP time to transmit all best effort downlink traffic through HCCA will improve even more the performance of the proposed HHE-CS mechanism. Two different scheduling algorithms have been studied for RT services as well as four different algorithms for best effort transmissions. The analysis of these different techniques has shown that, while a scheduling algorithm based on the arrival time of the video frames is the best one to fulfill the user QoS, in the presence of stations operating at different transmission modes an algorithm that takes into account the transmission rate of each station achieves better performance.

\section{ACKNOWLEDGMENT}

This work has been partially funded by CICYT (Spanish National Science and Technology Council) and the FEDER program of the European Commission under the project TEC2005-08211-C02.

\section{REFERENCES}

[1] J. Luo, R. Mukerjee, M. Dillinger, E. Mohyeldin, E. Schultz, "Investigation of Radio Resource Scheduling in WLANs Coupled with 3G Cellular Network", IEEE Communications Magazine, June 2003.

[2] IEEE 802.11e, "Wireless LAN Médium Access Control (MAC) and Physical Layer (PHY) specifications. Amendment 8 Medium Access Control (MAC) Quality of Service Enhancements", 2005.

[3] C. Sunghyun, J. del Prado, S. Shankar, S. Mangold, "IEEE 802.11e contention-based channel access (EDCF) performance evaluation," in Proc. IEEE ICC, vol. 2, pp. 1151-1156, May 2003.

[4] A. Grilo, M. Nunes, "Performance evaluation of IEEE 802.11e,", in Proc. IEEE PIMRC, vol. 1, pp. 511-517, Sept. 2002.

[5] C. Casetti, C. Chiasserini, "Improving fairness and throughput for voice traffic in 802.11e EDCA," in Proc. IEEE PIMRC, vol.1, pp. 525-530, Sept. 2004.

[6] A. Grilo, M. Macedo, M. Nunes, "A scheduling algorithm for QoS support in IEEE802.11 networks," IEEE Wireless Communications, vol.10, pp. 36- 43, Jun 2003.

[7] J. Gozálvez y J. Dunlop, "System Performance and Adaptive Configuration of Link Adaptation Techniques in Packet-Switched Cellular Radio Networks", Computer Networks Journal (Elsevier), pp 404-426, 2006.

[8] P. Barford, M. Crovella, "Generating representative web workloads for network and server performance evaluation", Joint International Conference on Measurement and Modeling of Computer Systems, June 1998.

[9] O. Lázaro, D. Girma and J. Dunlop, "H.263 video traffic modelling for low bit rate wireless communications", in Proc. IEEE International Symposium on Personal, Indoor, and Mobile Radio Communications, pp. 2124-2128, Sept. 2004. 